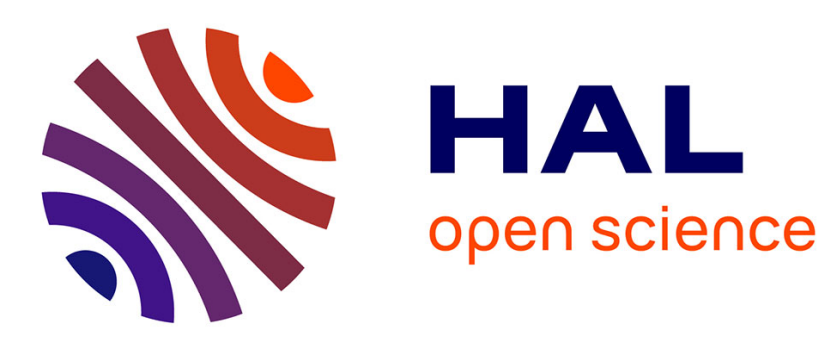

\title{
Dynamic Modeling of a Disturbance in a Multi-agent System for Traffic Regulation (decembre 2002)
}

\author{
Flavien Balbo, Suzanne Pinson
}

\section{To cite this version:}

Flavien Balbo, Suzanne Pinson. Dynamic Modeling of a Disturbance in a Multi-agent System for Traffic Regulation (decembre 2002). pp.20, 2002. hal-00017160

\section{HAL Id: hal-00017160 https://hal.science/hal-00017160}

Submitted on 17 Jan 2006

HAL is a multi-disciplinary open access archive for the deposit and dissemination of scientific research documents, whether they are published or not. The documents may come from teaching and research institutions in France or abroad, or from public or private research centers.
L'archive ouverte pluridisciplinaire HAL, est destinée au dépôt et à la diffusion de documents scientifiques de niveau recherche, publiés ou non, émanant des établissements d'enseignement et de recherche français ou étrangers, des laboratoires publics ou privés. 


\title{
Dynamic Modeling of a Disturbance in a Multi-Agent System for Traffic Regulation
}

\author{
Flavien Balbo ${ }^{*}$, Suzanne Pinson ${ }^{\S}$
}

\begin{abstract}
Résumé
Cet article présente la modélisation d'une perturbation sur un réseau de transport. Le modèle proposé doit assurer la synthèse, l'évaluation et la mise à jour des informations disponibles afin de faciliter la tâche de l'opérateur assurant la surveillance $\mathrm{du}$ réseau. Pour atteindre cet objectif, nous proposons une modélisation formelle du concept de perturbation. Cette modélisation permet de capitaliser les connaissances disponibles au sein d'un poste de contrôle et d'assurer le suivi du processus en temps réel. Nous proposons également une représentation multi-agent d'un incident permettant l'intégration du traitement d'une perturbation au sein de l'activité d'un réseau de transport.
\end{abstract}

Mots-clefs : Système d'aide à la décision, système multi-agent, transport.

\begin{abstract}
This paper presents the modeling of a disturbance on a public transportation line. The proposed model allows the synthesis, evaluation and update of available information in order to help human regulators in their monitoring task. It begins with a formal modeling of the disturbance concept. This modeling makes it possible to capitalize the knowledge available within a monitoring station and to follow up the evolution of the disturbances in real time. The paper goes on to propose a multi-agent representation of an incident allowing the integration of the disturbance processing within the activity of a network system.
\end{abstract}

Key words: Decision support system, multi-agent system, transport system.

\footnotetext{
* LAMSADE, Université Paris-Dauphine, \{balbo,pinson\}@lamsade.dauphine.fr

§INRETS - GRETIA, balbo@inrets.fr
} 


\section{Introduction}

Over the last few years, with the increase of the number of passengers and lines number, transportation network management has become more and more complex. This change in scale of network activity has to be accompanied by technological changes to the management system. These changes are necessary for bus networks, as opposed to guided transportation (train and subway [POM 96]), since vehicles move in urban traffic. For public transportation networks, the main efficiency criteria of their activity is their commercial speed. In France [Col 94], the commercial speed average is $15 \mathrm{~km} / \mathrm{h}$ whereas the ideal speed is about $22 \mathrm{~km} / \mathrm{h}$. For UTP (Union des Transports Publics) a $2 \mathrm{~km} / \mathrm{h}$ increase represents a profit of $0.3 \mathrm{~m} €$ for all the French provincial networks taken together. These economic studies underline the importance of an increase in speed.

As urban environment density increases, management techniques classically used by bus network regulators (the staff in charge of monitoring the bus network) became obsolete. While adapted decision support systems have been created for train [LIN 98] or subway [BRE 97] networks, the bus regulators have no decision support system adapted to their needs. A variety of approaches have been used to model urban transportation networks (Operation Research methods, fuzzy theory, neural networks, genetic algorithms, intelligent agents). A review of the literature can be found in [BAL 00] [BAL 01] [NII 01]. Regulators use systems known as Automatic Vehicle Monitoring systems (AVM) ([KHO 91] [MOR 91]), that were developed in order to better ensure the success of the transportation plan. An AVM allows the management of vehicles located by sensors. AVM compares the actual positions of vehicles (captured by the sensors) with their theoretical positions in order to provide the regulator with an overview of the routes. In this way the regulator can see whether the vehicles are running ahead of timetables or are running late. By comparing the theoretical information with the real one, the AVM system tries to detect delays and advances of buses on the network.

The use of an AVM is the first step to the computerization of the transportation network activity. However this system is limited to coping with disturbances linked to unanticipated demands and to traffic conditions. Moreover, the collecting and shaping of data are insufficient to help regulators. Some research proposes solutions for particular points (management of connections [BOO 92], real-time schedule management [LI 91]), but no system is able to manage all the difficulties dealing with the management of a bus network.

The aim of our decision support system named SATIR (Système Automatique de Traitement des Incidents en Réseaux) is to propose an adapted answer to the real-time problem of bus network management. We propose to use the multi-agent paradigm for SATIR. Remember that a multi-agent approach is well adapted to model bus network activities where autonomous entities, called agents, interact with each other in an open, 
uncertain, dynamic environment [WEI 99]. The autonomy of a multi-agent system and its ability to adapt and react are essential in the field of transportation where the environment is dynamic and uncertain. Most research has taken advantage of this fact ([FUN 98] [GOL 99] [ZHU 98]). The multi-agent paradigm makes it easier to solve the difficulties of network management in real time: managing the inconsistencies of the data of the sensors that locate the vehicles, assessing a disturbance according to its context, proposing feasible solutions. This article presents the part of SATIR dealing with the evaluation of disturbances in the context; the other part of SATIR is reported in ([BAL 00] [BAL 01].

The first section presents the concept of disturbance and our multi-agent model of a transportation network. The second section presents the formal model of a disturbance, explains how changes are measured and shows how the disturbance model is integrated into our multi-agent system. The third section gives initial results; the last section proposes a conclusion.

\section{The Needs for a Decision Support System}

\subsection{The disturbance diagnosis context}

As explained above, regulators use an AVM system to detect disturbances on the network, i.e., information about advances and delays of vehicles. Some AVM systems propose more sophisticated detections like detection of the delay on the next departure. However, this disturbance diagnosis process has several limitations:

- Lack of a global vision: The splitting up of monitoring by line and the high number of the lines to be monitored (each regulator tracks 13 lines with 5 to 20 buses running during the day) prevent global management of the network.

- Lack of space-time dimension: Primary alarms on the advance/delay of each bus provide too instantaneous a picture of line conditions. Monitoring all these alarms in their space-time development is almost impossible and leads to extra work for regulators.

Each regulator has to monitor more lines than he materially can see on his screen. The regulators first activity is to choose which lines to observe (for the transportation network under study, each regulator can handle 3 lines out of 13). This complex process involves various information sources (the AVM system for real-time information, theoretical timetables, information coming from drivers or from other regulators, etc.) [CAR 97]. An experimented regulator uses his knowledge of the line structure (the position in the city, the presence of difficult areas) and of the demand structure to determine the most critical lines according to the schedule. Since this kind of information is less available to novice regulators, they are less efficient in solving disturbance problems. 
As soon as a disturbance is chosen by the regulator, he has to complete his knowledge of the problem. This process is complex because disturbances evolve independently along three axes [SCE 98]:

- Time: this axis measures the seriousness of a disturbance according to the timetable. For example, a stop near a university should be monitored with timetables corresponding to arrivals and departures of students.

- Space: this axis measures the seriousness of a disturbance according to its position on the network. For example, some locations are known to be critical. A disturbance at these locations is more difficult to manage.

- Shape: this axis measures the consequences a disturbance may have on the network activity. For example, a vehicle running for its last journey is less critical than if it has to operate a connection.

To determine its importance, a disturbance must be evaluated according to these three axes. For example, a vehicle having off-peak hour difficulties in a suburb (a disturbance that is not critical a priori) may cause a real problem if bus frequency is such that users have to wait a long time for the next bus.

These three criteria underline the difficulty of the regulation task. The regulator has not only to establish a diagnosis on the current state problem, but also to consider its possible evolution along these three axes.

We have designed a dedicated decision support system to answer these problems. It takes into account the data coming from the existing information system (AVM system) and the environment characteristics that are as follows:

- Open environment: vehicles "appear" and "disappear" from the information system according to their activity or the regulator's needs.

- Uncertain environment: in most networks, vehicles are located through sensors that provide information that may be incomplete (a sensor breaks down), or uncertain (sometimes the quality of the data is poor).

- Dynamic environment: information concerning the location of vehicles is collected every 40 seconds (in our network) and represents a large data stream.

A multi-agent system has been defined to manage the transportation network under normal conditions (network monitoring, dynamic schedule management, data inconsistencies management [BAL 01]). To allow the appropriate management of available information, we propose to model the disturbance process in a dynamic way, from the beginning to the end of the disturbance process. In order to manage and simulate the network under normal and disrupted conditions, we have integrated our disturbance model in the multi-agent system. 


\subsection{A multi-agent system (MAS) to model network activity}

As said above, the regulators need a new decision support system in order to: 1) diagnose disturbances on the bus lines (buses running late, running early, etc.), 2) detect inconsistency in positioning data sent by buses to the central regulator, 3) dynamically compute schedules, and 4) monitor and process disturbances.

Very few models are based on the multi-agent paradigm because one of the difficulties in both the design and the understanding of MASs comes from the lack of central controls and the ensuing conflicting, uncertain, incomplete and delayed knowledge coming from the agents. Lind et al. used the multi-agent approach for transportation scheduling and simulation in a railroad scenario [LIN 98]. Brezillon has designed a simulator to help human regulators of the Parisian subway [BRE 97]. However, these systems present several drawbacks: they are mostly simulation systems that are not integrated into the decision support system, and they are not directly fed with real-time data coming from vehicle sensors.

In order to detect the delay of a vehicle in the network diagnosis process, the time when the bus is theoretically due has to be compared with the current time. Timetable management involves three steps: 1) making up the theoretical timetables; 2) monitoring the network activity (modifying the timetables according to where the vehicles actually are); 3) managing the inconsistencies of the data from the sensors that locate the vehicles.

In order to ensure the three functions, we propose two categories of agents as part of our multi-agent system:

- the STOP agents, that represent the theoretical structure of the network (organized in lines and routes) and calculate the theoretical timetables.

- the BUS agents, that represent the dynamic part of the network. Every BUS agent is the abstraction of an actual vehicle running on the transportation network and reports its movements to the STOP agents.

We have chosen to allow the agents to compute the theoretical timetables themselves in order to ensure that the multi-agent system is autonomous. The STOP agents have the knowledge used by the graph makers (traffic problems and passenger flow) to make up a timetable. This knowledge is also used in the assessment process and in the search for solutions to a disturbance Ten minutes before a vehicle depart, the STOP agents compute its timetable, taking into account the time of the day.

When a vehicle passes at a stop on the actual network, a warning message is sent from the BUS agent to the STOP agent concerned. The STOP agent updates its timetable by removing this vehicle from the list of vehicles due. A STOP agent which does not receive any message detects an anomaly and triggers the disturbance processing presented in this paper. 
In most multi-agent systems, the agents communicate in order to find skills or information that they need to achieve their goal. But in the domain of urban traffic control, because the receiver of the message is often identified according to its position, the sender does not always know the name of its receivers. For example, when a bus has to contact its nearest bus, it does not know its identification. We have designed an original communication model, called ESAC, that addresses this type of problem. The ESAC model proposes to use the environment as an active and intelligent entity that can send the right information to the right agent at the right time. More details can be found in ([BAL 00], [BAL 99]).

\section{Disturbance Modeling}

In this section, we present our modeling of a disturbance process in a transportation information system and its integration within our multi-agent model.

\subsection{Definition of a disturbance}

A transportation information system is a set of information where the access points are the vehicles and the stops. For instance, the identification of a vehicle is used to access the driver timetables. From this point of view, a disturbance is a subset of information network that has to be isolated and analyzed.

We have formalized a disturbance model, called "Incident model", in order to:

1. Search for and collect information necessary for regulators to solve the problem. This is a necessary step for regulator analysis and the information constitutes the decision context [BRE 99].

2. Dynamically update this knowledge over time: we propose a formal modeling of the information sets and the way they change according to changes on the network.

3. Summarize relevant information: we propose several measures that are based on the comparison of the relative changes in the information sets.

Whereas the AVM systems propose an instantaneous image of the network state that regulators have permanently to analyze, our system integrates data updates in the disturbance model allowing an incident to be analyzed from its beginning to its end.

The first goal of the Incident model is to collect information that is relevant to the problem under study and to reconstruct the context of the decision.

Describing a disturbance using the delay of a vehicle is not sufficient. For example, a vehicle may be running late, but the distance between the previous and the following vehicles is preserved. In this case, a regulator will not take the disturbance into account. He will be more interested in a vehicle with a shorter delay, but which leads to an imbalance along the line. To measure qualitatively the importance of a delay, we have taken into account its consequences on the activity of the network. 
For this purpose, we have defined three information sets, also called areas:

- a Successor area: This area brings together all the stops waiting for the successor of the late bus, it measures the risk assessment of a bus train (the late vehicle is caught up by the following one).

- a Critical area: This area brings together all the stops where the vehicle is late, it measures the risk assessment of a gap (the late vehicle is left behind by the preceding bus).

- a Predecessor area: This area brings together all the stops where the late vehicle is due but not yet late, it measures the risk assessment of a gap (the late vehicle is left behind by the preceding bus).

By drawing a distinction between the Successor and Critical areas, it is possible to compare incidents in terms of seriousness. For two incidents with the same number of points between the late bus and its predecessor, the incident with the greatest number of stops in the Critical area is considered as the most serious.

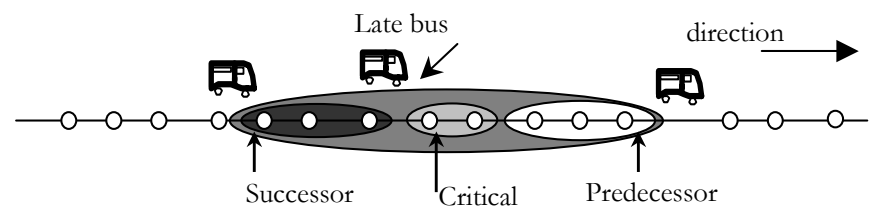

Figure 1: Representation of a disturbance

The set of these three areas constitutes the model that we call the Incident model (Figure 1).

The Incident model gathers in a single entity all the information necessary to manage a given disturbance. Since the regulators spend a large part of their time gathering information, this model will help them in their daily work

\subsection{Formal model of a disturbance}

\subsubsection{Some definitions}

In this section, the main definitions of the Incident model are introduced.

\section{Definition 1: Position relation}

A vehicle has to be identified according to its position in time and space.

Let $b$ be a bus; it is located at time $t$ at the stop $a_{i k}^{L}$ : 


$$
(b, t) \rightarrow a_{i k}^{L}=\operatorname{position}(b, t)
$$

$a_{i k}^{L}$ (nth. stop on route $i$ of line $L$ ) is the last stop where the vehicle was located.

\section{Definition 2: WaitedBus relation}

Because stops are grouped according to the vehicle they are waiting for (cf. Figure 1), it is necessary to identify this bus for each stop. This relation, called WaitedBus, is defined as follows:

$$
\text { WaitedBus }\left(a_{i k}^{L}, b, t\right) \Leftrightarrow\left(\operatorname{position}(b, t)=a_{i k^{\prime}}^{L}, k>k^{\prime}\right) \wedge\left(a_{i k}^{L} \in \operatorname{CurrentTrip}_{\mathrm{t}}(b)\right)
$$

A bus $b$ is expected at the stop $a_{i k}^{L}$ at time $t$.

The relation WaitedBus identifies the reference of vehicles that are due at the stop $a_{i k}^{L}$ at time $t$. The relation CurrentTrip represents the journey (a route with a schedule) of a bus $b$ running at time $t$.

Remark: this relation is checked by all the stops that are part of the current journey of the vehicle if their position is situated between the position of the last served stop and the position of the terminus.

\section{Definition 3: WaitingArea relation}

In order to define subsets of stops (called WaitingArea) waiting for a vehicle, the relation WaitedBus is used. A WaitingArea of a bus $b$ at time $t$ on route $i$ of line $L$ is defined as follows:

$$
\text { WaitingArea }(b, t)=\left\{a_{i k}^{L} \in A_{i}^{L} \mid \operatorname{WaitedBus}\left(a_{i k}^{L}, b, t\right)\right\}
$$

$A_{i}^{L}$ represents the set of stops on the same line and the same route. By convention, we define a neutral element WaitingArea (null, $t)=\varnothing$. The null constant represents the absence of any vehicles.

\section{Definition 4: Area modification relation}

The aim of our model is to study the evolution of a disturbance over time. To allow the follow-up of the process, we define an area according to the preceding state of the and to its modifications.

Let $b$ be a bus and $t, t^{\prime} \in \mathrm{T}\left(t<t^{\prime}\right)$ such that:

$$
\text { Position }\left(b, t^{\prime}\right)=a_{i k^{\prime}}^{L} \text { and position }(b, t)<\text { position }\left(b, t^{\prime}\right)
$$

Then: WaitingArea $\left(b, t^{\prime}\right)=\left\{a_{i k}^{L} \in\right.$ WaitingArea $\left.(b, t) ; k \geq k^{\prime}\right\}$

Each move of a vehicle between time $t$ and $t^{\prime}$ dynamically removes stops served between $\mathrm{t}$ and $\mathrm{t}^{\prime}$ from WaitingArea $(b, t)$. 


\section{Definition 5: WaitingAreaNextBus relation}

To model a disturbance, we need to know the stops a particular vehicle (the late bus, its following one or the preceding one) has to serve (which will not be served by another vehicle before its passage). This set (called WaitingAreaNextBus) is built using the difference between the WaitingArea sets of the two vehicles concerned.

WaitingAreaNextBus $(b, t)=\left\{a_{i k}^{L} \in\left(\right.\right.$ WaitingArea $(b, t)$ - WaitingArea $\left.\left.\left(b_{p}, t\right)\right)\right\}$

if $b$ has no successor, $b_{p}=$ null.

Remark: the set WaitingAreaNextBus $(b, t)$ will be noted ZonePb $(b, t)$ in the rest of this article.

These preliminary definitions are useful to define the conditions necessary to form the stop sets that compose the Incident model.

\section{Definition 6: CriticalArea relation}

The critical area of a bus $b$ at time $t$ on route $i$ of line $L$ is defined as follows:

$$
\text { CriticalArea }(b, t)=\left\{a_{i k}^{L} \in \operatorname{ZonePb}(b, t) ; \text { Delay }\left(a_{i k}^{L}, b, t\right)\right\}
$$

\section{Definition 7: PredecessorArea relation}

When the CriticalArea $(b, t)$ exists, PredecessorArea of a bus $b$ at time $t$ on route $i$ of line $L$ is defined as follows:

$$
\text { PredecessorArea }(b, t)=\left\{a_{i k}^{L} \in \operatorname{ZonePb}(b, t) \vdots \neg \text { Delay }\left(a_{i k}^{L}, b, t\right)\right\}
$$

CriticalArea $(b, t)$ groups the stops of $\operatorname{ZonePb}(b, t)$ for which the bus $b$ is late at time $t$. PredecessorArea $(b, t)$ is defined as the set of stops of CriticalArea $(b, t)$ and ZonePb $(b, t)$ at time $t$.

\section{Definition 8: SuccessorArea relation}

When the CriticalArea $(b, t)$ exists, SuccessorArea of a bus $\mathrm{b}$ with regard to its successor $b^{\prime}$, at time $t$ on route $i$ of line $L$, is defined as follows:

$$
\text { SuccessorArea }\left(b, b^{\prime}, t\right)=\operatorname{ZonePb}\left(b^{\prime}, t\right)
$$

This area is defined according to the successor of the bus responsible for the disturbance.

\section{Definition 9: Incident relation}

The Incident relation is the grouping of the areas described above:

$$
\text { Incident }\left(b, b^{\prime}, t\right)=\left\{\text { CriticalArea }(b, t) \text {, PredecessorArea }(b, t) \text {, SuccessorArea }\left(b, b^{\prime}, t\right)\right\}
$$

Incident $\left(b, b^{\prime}, t\right)$ dynamically change according to time, that means that each area varies according to buses movements on the network. 


\subsubsection{Example of follow-up of a disturbance}

This part illustrates how our model is used to follow the progression of a disturbance on a transportation system.

Our model is used to verify the coherence of stops transition from one step to another. We verify that the transitions of stops between areas are in agreement with events that have caused them.

The initial situation is the following one: the bus $b_{r}$ is late and responsible for the disturbance, the bus $b_{p}$ precedes it and $b_{s}$ succeeds it. For each of these vehicles, there is a set Zone $\mathrm{Pb}\left(b_{x}, t\right)$ with $\left(b_{x}=b_{p}\right.$, or $b_{r}$ or $\left.b_{s}\right)$.

We describe the kinematics of a disturbance in the case where there is no change in the order of vehicles (no appearance or disappearance):

\section{Creation of an Incident.}

At time $t$, an Incident is created if:

$$
\exists a_{i k}^{L} \in \operatorname{ZonePb}\left(b_{r}, t\right) \wedge \text { delay }\left(a_{i k}^{L}, b_{r}, t\right) \wedge \neg\left(\exists \text { CriticalArea }\left(b_{r}, t\right)\right)
$$

An Incident is created if a stop that is waiting for a vehicle (not already concerned by a disturbance) detects a delay with respect to the schedule.

According to the previous definitions:

CriticalArea $\left(b_{r}, t\right)=\left\{a_{i k}^{L} \in \operatorname{ZonePb}\left(b_{r}, t\right):\right.$ Delay $\left.\left(a_{i k}^{L}, b_{r}, t\right)\right\}$

PredecessorArea $\left(b_{r}, t\right)=\left\{a_{i k}^{L} \in \operatorname{ZonePb}\left(b_{r}, t\right) \backslash \neg\right.$ Delay $\left.\left(a_{i k}^{L}, b_{r}, t\right)\right\}$

SuccessorArea $\left(b_{r}, b_{s}, t\right)=\operatorname{ZonePb}\left(b_{s}, t\right)$

The Successor area can be empty if:

- The Successor of $b_{r}$ is unknown: it may occur if journeys are spaced out over large time intervals. In our multi-agent organization, only buses having announced their departure by asking for the creation of a schedule shortly before the departure are known. If an incident is detected whereas the schedule for the following bus has not yet been calculated, this vehicle is unknown.

- ZonePb $\left(b_{s}, t\right)$ is empty: it may occur if two vehicles form a bus train (i.e., convoy), no stop is waiting for the successor.

\section{Change of vehicle position}

As described before, the content of a stop area is modified by the movement of the vehicles that surround it. 
Thus the contents of CriticalArea $\left(b_{r}, t\right)$ and PredecessorArea $\left(b_{r}, t\right)$ are modified according to the movements of $b_{r}$ and $b_{p}$ (according to the definition of ZonePb) while SuccessorArea $\left(b_{r}, b_{s}, t\right)$ depends on $b_{r}$ and $b_{s}$.

The creation of the sets depends on the following events at time $t^{\prime}$ : the movement of the bus $b_{p}$, the movement of the bus $b_{r}$, the movement of the bus $b_{s}$.

- The movement of the bus $b_{p}$ :

position $\left(b_{p}, t^{\prime}\right)=a_{i k^{\prime}}^{L}$,

We distinguish four possibilities for stops $a_{i k}^{L}$ of $\operatorname{ZonePb}\left(b_{p}, t\right)$ verifying condition $k$ $\leq k^{\prime}$ :

1. WaitedBus $\left(a_{i k}^{L}, b_{r}, t^{\prime}\right) \wedge \neg$ delay $\left(a_{i k}^{L}, b_{r}, t^{\prime}\right) \Rightarrow a_{i k}^{L} \in \operatorname{PredecessorArea}\left(b_{r}, t^{\prime}\right)$

Stops have a normal transition, from one waiting zone to the next.

2. WaitedBus $\left(a_{i k}^{L}, b_{r}, t^{\prime}\right) \wedge$ delay $\left(a_{i k}^{L}, b_{r}, t^{\prime}\right) \Rightarrow a_{i k}^{L} \in \operatorname{CriticalArea}\left(b_{r}, t^{\prime}\right)$

$b_{p}$ itself is late and the delay of $b_{r}$ is such that the new stops are placed directly in the critical area.

3. WaitedBus $\left(a_{i k}^{L}, b_{s}, t^{\prime}\right) \Rightarrow a_{i k}^{L} \in \operatorname{SuccessorArea}\left(b_{r}, b_{s}, t^{\prime}\right)$

Two vehicles are sufficiently close (bus train) so that stops are waiting $b_{s}$ at time $t^{\prime}$.

4. WaitedBus $\left(a_{i k}^{L}, b_{y}, t^{\prime}\right) \wedge \operatorname{position}\left(b_{y}\right)=a_{i k^{\prime \prime}}^{L} \operatorname{position}\left(b_{s}\right)=a_{i k^{\prime \prime}}^{L}: k^{\prime \prime}<k^{\prime}$

$$
\Rightarrow a_{i k}^{L} \in \operatorname{ZonePb}\left(b_{y}, t^{\prime}\right)
$$

Three vehicles are sufficiently close that stops are waiting for a vehicle that is not concerned by the disturbance (this bus is noted $\mathrm{b}_{\mathrm{y}}$ and situated after $b_{s}$ ).

- The movement of the bus $b_{r}$ :

position $\left(b_{r}, t^{\prime}\right)=a_{i k^{\prime}}^{L}$,

We distinguish two cases for each ZonePb's stop $a_{i k}^{L}\left(b_{r}, t\right)$ verifying $k \leq k^{\prime}$.

1. WaitedBus $\left(a_{i k}^{L}, b_{s}, t^{\prime}\right) \Rightarrow a_{i k}^{L} \in \operatorname{Successor}\left(b_{r}, b_{s}, t^{\prime}\right)$

Stops have a normal transition, from one waiting area to the next.

2. WaitedBus $\left(a_{i k}^{L}, b_{x}, t^{\prime}\right) \wedge$ position $\left(b_{x}\right)=a_{i k^{\prime \prime}}^{L} \operatorname{position}\left(b_{s}\right)=a_{i k^{\prime \prime \prime}}^{L} \mid k^{\prime \prime}<k^{\prime}$

$$
\Rightarrow a_{i k}^{L} \in \mathrm{ZonePb}\left(b_{x}, t^{\prime}\right)
$$

Vehicles $b_{s}$ and $b_{r}$ are sufficiently close (bus train) that stops are waiting for another noted bus $b_{x}$ located after $b_{s}$ at time $t^{\prime}$. 
In initial $\operatorname{ZonePb}\left(b_{r}, t\right)$, stops can also move from PredecessorArea $\left(b_{r}, t\right)$ to CriticalArea $\left(b_{r}, t\right)$ :

$a_{i k}^{L} \in$ PredecessorArea $\left(b_{r}, t\right) \wedge$ delay $\left(a_{i k}^{L}, b_{r}, t^{\prime}\right) \Rightarrow a_{i k}^{L} \in$ CriticalArea $\left(b_{r}, t\right)$

- The movement of the bus $b_{s}$ :

This case is the easiest one because the served stops are no longer taken into account by the model.

\subsection{Modeling the consequences of a disturbance}

The Incident model is used to bring together information on a disturbance and to organize it automatically according to how it is linked to the problem. In this section, we show how to use this knowledge to give the regulator a qualitative disturbance evaluation. We propose to measure the risk of a bus train and the risk of a gap. We have defined three indicators based on the comparison of the difficulties for the vehicles concerned to progress. The proposed heuristic uses the theoretical difficulties that a vehicle may meet on a network subsection.

A data table is associated with each point of the network for each period of time (classified from 0 (fluid) to 2 (heavy traffic)). These data are used to define the theoretical state of traffic and of passenger demand, and it is these theoretical data that are used to compute schedules. Nevertheless, the disturbance dynamics may lead to a gap between supply and demand; when the late vehicle has to answer a strong demand, the following one deals with a weaker one. That is why we suggest to modify the initial values of the theoretical passenger flow evaluation according to their link to the disturbance. The value must be reduced for the stops in the Successor area and increased for the stops in the Critical and Predecessor areas. After various observations, we have defined a progression indicator (Ip) so as to put in perspective the size of the passenger flow in the various areas.

Let $\mathrm{y}$ be an area, $\mathrm{x}$ the number of stops in this area, $M_{p}(i)$ the traffic evaluation of the nth stop of the area $y$ and $M_{v}(i)$ the passenger flow evaluation of the nth stop of the area $y$. The progression coefficient measures the disturbance state compared to an average situation (i.e., $M_{p}(i)$ and $M_{v}(i)$ are equal to one for each $i$ ):

$$
\text { Progression_Coefficient }(x, y)=\frac{\sum_{i=1}^{x}\left[\left(M v(i)^{*}(1+I p(y))\right)+M p(i)\right]-2 x}{x}
$$

If this coefficient is positive, it means that the bus may slow down in the area, otherwise, it means that the area may allow the bus to make up for lost time.

The comparison of the area progression coefficients is used to evaluate the risks linked to a disturbance. Let $z 1$ be the SuccessorArea, $z 2$ be the CriticalArea and $z 3$ be 
the PredecessorArea; we define two measures: the risk of a bus train (Rtdb) and the risk of a gap (Rlac).

1) The first vehicle may be slowed down but not the following one: If the first vehicle is the late bus, then the risk of a bus train can be computed. If the first vehicle is the predecessor of that bus, then a possible modification of the disturbance is identified. The actual disturbance may disappear and the predecessor may create a new disturbance. To calculate the bus train risk, we compute the difference between the Successor area progression coefficient and the Critical area progression coefficient.

$\mathbf{R t d b}=[$ Progression_Coefficient $(\min , z 2)$ - Progression_Coefficient $(\min , z 1)] / x$

Since an Incident moves on the network, the number of stops in each area varies from one evaluation to the other. Furthermore, to obtain comparable measures, the number of stops must be the same in the two areas. Consequently, we have chosen to calculate the progression coefficients for a number of stops (noted min) equal to the minimum number of stops in the two areas. The greater the number of stops (noted $x$ ) contained in the SuccessorArea, the lower the risk.

- The first vehicle may accelerate but not the following one: If the first vehicle is the predecessor of a late bus, the risk of a gap is identified. If the first vehicle is the late bus, then a possible disturbance modification is identified. The actual disturbance may disappear and the successor may create a new disturbance. To calculate the risk of a gap, we add the progression coefficient of the CriticalArea to that of the PredecessorArea. The gap risk takes into account the number of stops between the late bus and the previous vehicle.

Let $x$ be the number of stops in the CriticalArea and $y$ the number of stops in the PredecessorArea.

Rlac $=[$ Progression_Coefficient $(x, z 2)+$ Progression_Coefficient $(y, z 3)] *(x+y)$

- Two areas change in the same way (two vehicles may accelerate or slow down): the analysis is more difficult and depends on the study of the respective speeds of vehicles.

\subsection{Integrating the disturbance model into the multi-agent system}

This section describes the agents we have defined and the organization needed to model dynamically the disturbance process within our multi-agent network modeling.

The initial organization of the multi-agent system (in lines and routes) must be completed by a new organization for the agents concerned by a disturbance (Fig. 2). We propose to dynamically create a hierarchy where each level corresponds to an information synthesis. We need two new types of agents:

- An AREASTOP agent: Each of them manages STOP agents according to the set presented before (Fig 1). It collects, updates and synthesizes useful information. For example, it calculates passenger flow for the STOP concerned. 
- An INCIDENT agent: It synthesizes information from AREASTOP agents. Because it collects all useful information such that the solutions that are proposed by the BUS agents, it is the interface between regulators and disturbances. For its synthesis activity, the INCIDENT agent computes the risk coefficients presented in section 2.4.

The lowest level of the hierarchy concerns the STOP agents, that constitute the elementary entities, i.e., for each stop, basic information such as theoretical traffic difficulty or size of user flows. The middle level concerns the STOPAREA agents that makes an initial information synthesis. At this level the progression difficulties and the demand level linked to a stop set are computed. The INCIDENT agent represents the top of the hierarchy where risks are calculated. Consequently it is the highest level of the information synthesis.

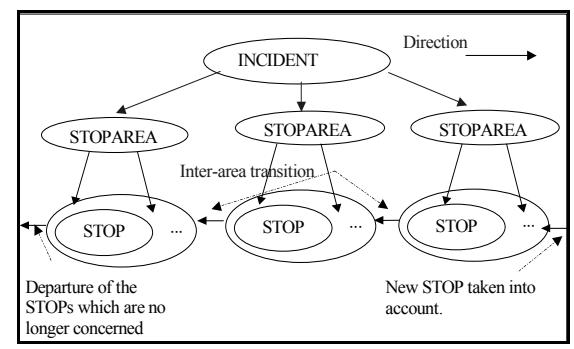

Figure 2: Dynamic organization

This organization is dynamic because the STOP agents move both from one area to the other within the hierarchy, and from and towards the outside of the organization, according to traffic direction.

The process of hierarchy creation takes place in three stages:

- Stage 1: The STOP agent that detects a delay creates the INCIDENT and STOPAREA agents necessary to solve the problem.

- Stage 2: These newly created agents contact the STOP agents that are expecting the late bus or its following one, and send messages to the STOPAREA agent on which the STOP agent depends. Each STOP agent that receives a message sends messages to the STOPAREA agent on which it depends.

- Stage 3: The STOPAREA agent contacts the BUS agents concerned by the disturbance, the late vehicle and its following one, to inform them about the disturbance that has been detected.

Each disturbance depends on the network activity and mostly on the appearance and disappearance of the vehicles. As an example, let us consider a particular case study: the planned insertion of a new vehicle by a regulator. The new vehicle is inserted between the late bus and its following one. The fact that the new vehicle becomes the following bus of the late vehicle modifies the definition of the Successor area. The STOP agents 
that detect a modification of the next vehicle reference they are waiting for, contact the STOPAREA agent concerned to inform it about this event. This agent reacts by considering the new vehicle as the vehicle concerning its area. The STOPAREA agent sends a message to the STOP agents to tell them that they are not concerned anymore by the disturbance (the stops situated between the new successor and the former one).

\section{Implementation and Results}

A prototype has been implemented in $\mathrm{C}++$. In order to study the feasibility of our proposal, the prototype has been tested using real data from the Brussels Intercity Company network (STIB) recorded on tapes. The tests were carried out over 8 days and 300 incidents were assessed.

This section gives the initial results of our decision support system. First we detail two incident examples that were detected on the STIB network, then we explain the interface of the system.

\subsection{Disturbance assessment}

In this section, we propose a qualitative assessment of the evolution of a disturbance evolution. There are two incidents: the first characterizes the disturbance linked to a difficult section on the network, the second characterizes incidents relative to a vehicle. For each incident (Figures 3 and 4), two graphs are presented (a and b) that show, according to time (measured in cycles), the evolution of the number of stops in an area and the evolution of the associated risk. The first two graphs (Figure 3a and 4a) show the changes to the Successor and Critical areas and the risk of a bus train. The next graphs (Figures $3 \mathrm{~b}$ and $4 \mathrm{~b}$ ) show the changes to the Critical and Predecessor areas to the sum of these two areas and to the risk of a gap.

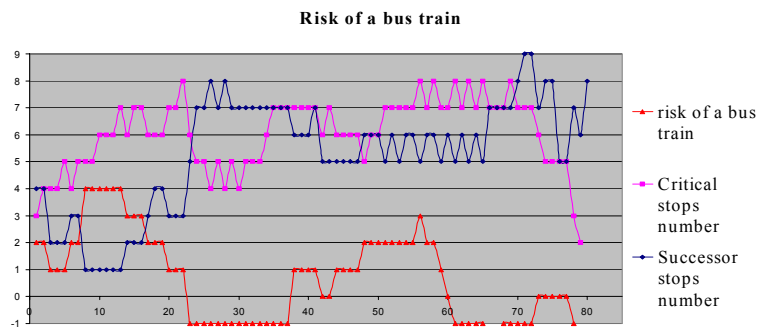

a) 


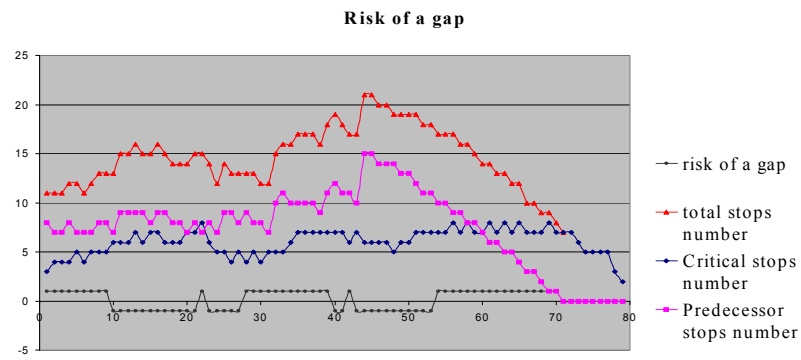

b)

Figure 3: Risks analysis of a disturbance

- Analysis of a difficult network section: Figure 3 shows a vehicle problem at a particular network section and its consequences. The late vehicle is stopped (creation of the disturbance, then increase in the number of stops in the Critical area from cycle 0 to cycle 20). Then its successor catches up with the late vehicle (increase in the risk of a bus train (Figure 3a)). After this, the late vehicle is freed (from cycle 22) and number of stops in the Critical area decreases considerably (from 8 to 5) before stabilizing at around 7 stops. The stops lost by the Critical area are gained by the Successor area and stabilize at around 6 .

The following vehicle does not manage to catch up the late bus. This constitutes a risk of transfer of the disturbance from the current late bus towards the following one. The study of the area changes linked to the risk of a gap confirms this tendency (Figure 3b)).

When the disturbance starts, the total number of stops starts to increase and the late vehicle falls further behind. Then the situation stabilizes. In other words, the initial situation was critical since the risk of a bus train and the risk of a gap were high, until it stabilized. Although the vehicle did not make up its delay, the disturbance is no longer critical (the risk of a bus train and the risk of a gap decrease).

- Analysis of an incident linked to a vehicle: Figure 4 shows that vehicle difficulties last throughout its activity. The number of stops in the Critical area shows two peaks that correspond to an increase in the vehicle delay (cycles 20-40 and 72-90 Figures 4 a) and $b$ )). The risk of a bus train and the risk of a gap are permanent. The number of stops in the Successor area is low and becomes null at the end of the disturbance. The total number of stops between the late bus and its predecessor increase constantly. In this case, this disturbance requires the intervention of the regulator because the risks relative to this disturbance are high. 
Risk of a bus train

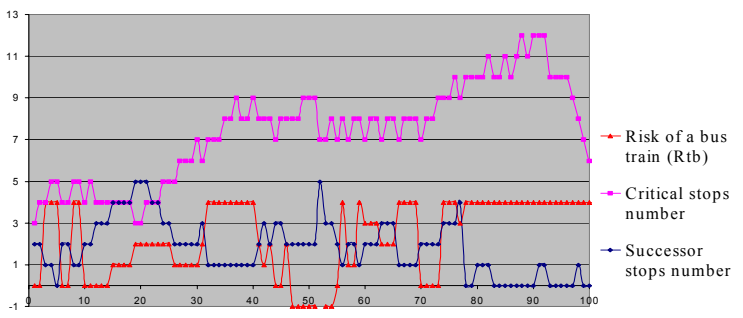

a)

Risk of a gap

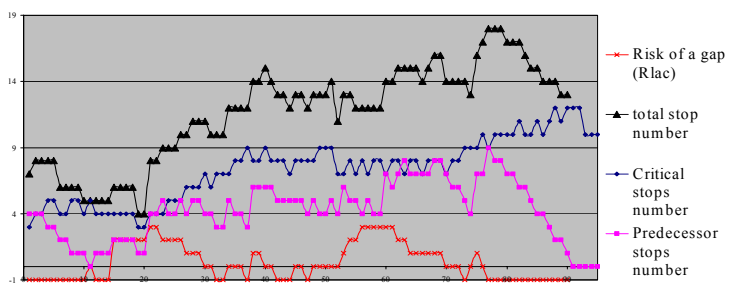

Figure 4: Risks analysis of a vehicle in a difficult situation

These two examples illustrate how our Incident model allows a sharper disturbance evaluation. Existing systems detect the vehicle delay but do not put the seriousness of these delays into perspective. Thanks to the Incident model, the regulators are able to know the real difficulties of the vehicle.

\subsection{Interface with the regulator}

The interface shown in Figure 5 is proposed to the regulators. This interface produces a summary of all the data concerning the problems on the network. It contains the list of disturbances detected on the network, their identification, their location, their starting time, their seriousness, the identification of the bus involved. 


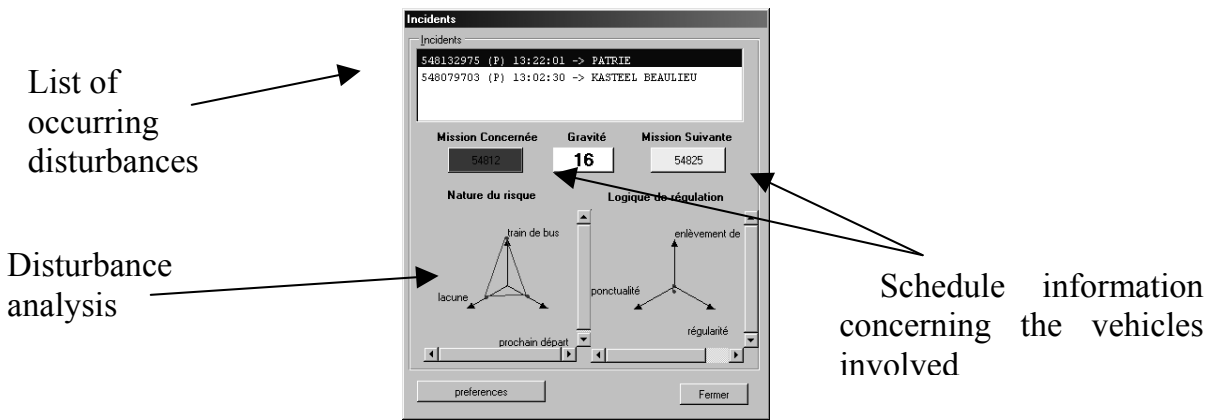

Figure 5: The regulator interface of the network incident management system

One of the advantages of our system over the preceding ones is that the regulator has no longer to choose the lines to be monitored. The interface guides him towards lines with problems. For every disturbance the regulator has access to its description by clicking on the reference of the late vehicle (here 54825) and of the following one. The information concerns its timetable. The assessment of the risks is given by a triangle, the distortion of which informs the regulator of the relative seriousness of the risks. If the letter (P) appears in the lists of disturbances, then some solutions have been proposed to reduce the problem (see [BAL 00] for a detailed presentation of solution formation).

\section{Conclusion}

In this paper, we have presented a decision support system that offers a global approach to the regulation task on a transportation network. It detects disturbances, assesses them and look for a solution to reduce the problems resulting from a vehicle being late. The experience gained from the development effort of our system supports the proposition that the multi-agent paradigm is an appropriate framework for transportation network modeling and simulating.

The originality of our approach is the dynamic modeling of a disturbance process from its beginning to its end and it integration in a multi-agent system. We have defined a model, called the Incident model, that allows information synthesis that is useful for decision making. Through this model, knowledge relative to the network structure and knowledge relative to the network dynamics (stored in STOP agents and in BUS agents respectively) are gathered within a single entity. This entity allows the follow-up of the disturbance over space and time; it is deleted when the disturbance is solved.

We have defined two measures of risk linked to a disturbance. These measures are based on the study of a priori progression difficulties of vehicles concerned by the disturbance and take into account the intrinsic dynamics of a disturbance. 
The system has been tested using real data from the Brussels Intercity Company network (STIB). It presents several advantages over existing ones: more disturbances are detected and more information is provided to the regulators in terms of risk (risk of a bus train, risk of a gap, seriousness of a disturbance). This information allows them to take quicker and more accurate decisions. More research has to be done in several directions: 1) adding more knowledge to each agent of the system and fully testing and validating the system, 2) considering using our system as a simulator in order to validate new timetables and to serve as a training tool, 3) adding an economic planning module to propose a solution to regulators according to the risk of the problem getting worse.

\section{References}

[BAL 99] BALBO F., "A model of Environment, active support of the communication", in Proceedings AAAI Workshop on Reasoning in Context for AI Application. AAAI Technical Report WS-99-14, pp 1-5, AAAI Press, Menlo Park, CA, Orlando 1999.

[BAL 00] BALBO F., "ESAC: un Modèle d'Interaction Multi-Agent utilisant l'Environnement comme Support Actif de Communication. Application à la gestion des Transports Urbains", PhD Thesis, Université Paris IX Dauphine, January 2000.

[BAL 01] BALBO F., Pinson S., "Towards a Multi-Agent Modelling Approach for Urban Public Transportation Systems", in Omicini A., Petta P., Tolksdorf R. (Eds) Engineering Societies in the Agents Worlds II, LNAI 2203,pp 160-174 Springer Verlag, 2001.

[BOO 92] BOOKBINDER J.H., DÉSILETS A., "Transfer Optimization in a Transit Network", Transportation Science, Vol. 26, n²,pp 106 - 118 May 1992.

[BRE 97] Brézillon P., Gentile C., SAker I., Secron M., "SART: a System for Supporting Operators with Contextual Knowledge" in Proceedings International and Interdisciplinary Conference on Modeling and Using Context (CONTEXT 97),pp 209 -222, Brazil 1997.

[BRE 99] BrÉzillon P., Pomerol J.C., "Contextual Knowledge and Proceduralized Context", in Proceedings AAAI Workshop on Reasoning in Context for AI Application. AAAI Technical Report WS-99-14, pp 16-20, AAAI Press, Menlo Park, CA, Orlando 1999.

[CAR 97] CARUSO M., "Observation du Poste de Travail de Régulateur dans un P.C. d'Autobus, le réseau de la STIB à Bruxelles" Internal Report, Institut National de Recherche sur les Transports et leur Sécurité (INRETS), October 1997.

[COL. 94] Co-authored, "Transports Urbains, Comment Augmenter la Vitesse Commerciale", In le Courrier des maires $n^{\circ} 4,1994$. 
[FUN 98] Funk P., Vierke G., BÜRCKert H.J, "A Multi-Agent Perspective on Intermodal Transport Chains", Dans DFKI Technical Memo,1998-06.

[GOL 99] Goldsmith Y., Phillips L.R., Spires S.V., "A Multi-Agent System for Coordinating International Shipping", Lecture Notes in Computer Science, vol. ${ }^{\circ}$ 1571, pp 91--104,1999.

[KHO 91] Khorovitch B.G., Catalano G., Höflinger P, Leprince M., "Aspects techniques et économiques de systèmes d'aide la décision", in Proceedings Transport Public 91, Union Internationale des Transports Publics, $49^{e}$ International Congress, Stockholm, 1991.

[LI 91] Li Y., Rousseau J.M., Wu F., "Real - Time Scheduling on a Transit Bus Route", dans collection: CRT Pub. No 768 ; DIRO Pub. No 771 (24p.), Avril 1991.

[LIN 98] Lind J., FISCHER K., "Transportation Scheduling And Simulation In A Railroad Scenario: A Multi-Agent Approach", DFKI Technical-Memo-1998-05.

[MOR 91] MorloK E., BruUn E., Blackmon B., "Advanced Vehicle Monitoring and Communication Systems for Bus Transit: Benefits and Economic Feasibility", Final Report September 91, Revised March 93, Office University Research and Training Program, Office of Technical Assistance Federal Transit Administration Washington, DC 20590.

[NII 01] NitTTyMÄKI J., PURSUla M., Special Issue: "Artificial Intelligence on Transportation Systems and Science", European Journal of Operational Research, volume 131, n², pp 229-308, 2001.

[POM 96] Pomerol J.-C., Roy B., Rosenthal-Sabroux C., "Developing an 'intelligent' DSS for the multicriteria evaluation of railway timetables, problems and issues", Journal of Decision Systems, Vol. 5, n 3-4, 249-267.

[SCE 98] ScEMAMa G., GaUdin E., "Informatisation de la décision dans l'exploitation du transport, atouts des technologies avancées du traitement de l'information", Recherche Transports Sécurité nº61, pp 53-72, October - November 1998.

[WEI 99] WeISS G., Multiagent Systems: a Modern Approach to Distributed Artificial Intelligence, MIT Press, 1999.

[ZHU 98] Zhu K., Ludema M.W., van DeR Heijden R.E.C.M., "Air Cargo Transport by Multi-Agent Based Planning" 33rd Hawaii International Conference on System Sciences, 1998. 